\title{
Understanding teenagers' personal contexts to design technology that supports learning about energy consumption
}

\author{
Katerina Avramides, Brock Craft and Rosemary Luckin
}

London Knowledge Lab, Institute of Education, University of London

Correspondence: 23-29 Emerald Street, London WC1N 3QS. Email:

K.Avramides@ioe.ac.uk

K. Avramides holds a BSc in Psychology and Artificial Intelligence from the University of Nottingham (2001), an MSc in Human Centred Computer Systems (2003) and PhD in Informatics (2009) from the University of Sussex. She worked as a Research Associate in the area of cognitive science and computational modelling at Carnegie Mellon University. Since completion of her PhD she has worked as a Research Fellow on several multi-disciplinary projects, in both psychology/education and technical development roles, at the University of Sussex and recently at the London Knowledge Lab. Current research interests include the design and development of technology enhanced learning (TEL) experiences, particularly to support higher order thinking skills, and the role of affect and motivation in learning.

B. Craft holds a BA in Photography from Columbia College (1992), an MS (with Distinction) in Human-Computer Interaction (2001) from DePaul University, and a PhD in Computer Science (2007) from University College London. He was co-director of TinkerLondon, a technology startup specialising in design consultancy on the Internet of Things (IoT) and is currently a Research Fellow at the London Knowledge Lab. He has worked on a variety of interdisciplinary research projects focused on user-centred design, involving clinical e-Science, tangibles for learning, Learning Design, and Technology-Enhanced Learning. He also lectures in Arts Computing. Current research interests are in design prototyping, sketching and design cognition, information design, and ubiquitous computing.

R. Luckin holds a BA (Hons.) First Class, in Computing and Artificial Intelligence, awarded in 1993 and a PhD in Cognitive Science awarded 1998. She has previously held the following posts at the University of Sussex: Pro-Vice-Chancellor (Teaching and Learning), Director of the Human Centred Technology research group, Reader in Computer Science and Artificial Intelligence, Director of Undergraduate Studies for Science and Technology. Prof. Luckin took up her current post as Professor of Learner centred design at the London Knowledge Lab, University of London in 2006. She has also acted as a Non-executive Director, British 
Educational Communications and Technology Agency, appointed by the Secretary of State for Education from 2006 to 2011. She has written over 50 peer reviewed journal papers and book chapters, a research monograph and numerous conference papers. Prof. Luckin has served as a member of funding panels for both EPSRC and ESRC and is on the editorial board of the Journal of Computer Assisted Learning (JCAL), the International Journal of Artificial Intelligence in Education (IJAIED), Interactive Learning Environments (ILE), and Research and Practice in Technology Enhanced Learning (RPTEL). Her research explores how to scaffold learning across multiple technologies, locations, subjects and times through the application of participatory methods to the development and evaluation of Technology for learning. This work is interdisciplinary and encompasses Education, Psychology, Artificial Intelligence and HCI. She has developed strong links with research users and beneficiaries that contribute to both current and future projects. The total value of the research grants funded to date is in excess of $£ 10$ million (with $£ 3$ million awarded to her home institution).

\begin{abstract}
Energy sustainability is prevalent in political and popular rhetoric and yet energy consumption is rising. Teenagers are an important category of future energy consumers, but little is known of their conceptions about energy and energy saving. We report on empirical research with two groups of teenagers. This is part of ongoing work to design learning technologies that support teenagers learn about personal energy consumption. In this paper we describe our analysis and methodology, which are shaped by the Ecology of Resources design framework (Luckin, 2010). Our findings informed the development of an Ecology of Resources model of the participants' personal context, which includes their world resources (people, tools, knowledge, skills, and environment) and their personal resources (conceptions, motivations and concerns around energy consumption). We discuss the range of methods we employed to understand learners' personal contexts. These findings contribute to our understanding of how to explore teenagers' personal contexts and have implications for the design of technology to support learning about personal energy consumption.
\end{abstract}

\title{
Introduction
}

Climate change and resource scarcity have brought energy sustainability to the forefront of environmental concerns. An important factor in shaping future patterns of energy consumption is the understanding of the problem by younger generations and their willingness to address it. Teenagers are not the principal decision-makers within the 
household, but they are well placed to have an impact, both by changing their own behaviour and by influencing others within the family, their social network and the public sphere (Larsson, Andersson \& Osbeck, 2010). We know little about teenagers' understanding and behaviours in relation to energy consumption. Some studies involving teenagers have presented a negative picture of their contribution to energy consumption (Thøgersen \& Grønhøj, 2010; BBC, 2006). We can, however, observe that studies among adults suggest a widespread lack of awareness of the energy intensity of different behaviours (Steg, 2008). There is also evidence that adults do not connect environmental issues that arise from energy use with individual consumer choices, and are not willing to adopt changes in behaviour that have high impacts but are inconvenient (Lorenzoni, Nicholsoncole, \& Whitmarsh, 2007; Whitmarsh, 2009).

This lack of awareness of the connection between individual actions and larger scale problems, and also of how individuals might act to make a difference, suggests the need to embed learning about why energy consumption is an issue, and how to mitigate the problems, in the everyday contexts in which energy related behaviour and choices are made. This helps learners apply knowledge about energy use to their personal actions, and understand the impact of their behaviour in terms of energy related issues. Key information about energy use and the problems that arise from it must, therefore, be personalised. Personalising information includes presenting it in such a way that learners are motivated to engage with it. The adaptability, flexibility, and portability of learning technologies make them well placed to create such learning experiences. However, to personalise the learning experience to teenagers we must identify teenagers' personal contexts, and tailor the technology to them. The Ecology of Resources design framework (EoR, Luckin, 2010) offers a three-phase process for identifying and modelling learners' contexts for the purpose of designing technology enhanced learning experiences. In this paper, we report on the application of the 
first phase of the EoR framework. We examine the methods we used to identify and model teenagers' contexts, we discuss the resulting model of teenagers' contexts in relation to personal energy consumption, and consider how this model informs the design of learning technologies in subsequent Phases of the EoR. The paper contributes to our understanding of the initial stages of designing learning technologies that are embedded in learners' personal contexts. Specifically, it contributes to understanding of: a) more generally, methods (within the context of the EoR) for engaging with teenagers to identify and model their personal contexts, and b) specifically, the design of learning technologies to support teenagers to learn about personal energy consumption.

\section{Methodology and research questions}

The Ecology of Resources design framework (EoR, Luckin, 2010) offers a process for working with participants that models and takes account of their context. The framework is inspired by a sociocultural philosophy of understanding and supporting learning (Vygotsky, 1978, 1986) and the notion of scaffolding, a process of providing support that is closely contingent on the learner's current understanding and skills (Wood, 1976, Luckin et al, 2011; Pea, 2004). The EoR provides a method for designing learning technology and/or learning technology use that considers the important relationship between the learner in their context and the learning that arises out of their interactions with their context. The EoR conceptualizes a person's context in terms of their interactions with the multiple resources of their world, for example, with the people, places, books, knowledge concepts and technologies they encounter; and in terms of their personal resources, such as their motivation and existing understanding. To support learning the design process seeks to effectively link the world resources that are most appropriate for a specific learning goal and to scaffold interactions between these resources and the learner, taking into account the 
learner's personal resources.

The EoR provides a method through which we first identify the world resources available to the learner and the processes and relationships that shape the learner's access to these. We also build an understanding of the learner and what they bring to the learning experience: their personal resources. The EoR also introduces the notion of filters to describe the artefacts that constrain a learner's access to resources, such as rules, regulations or physical boundaries. Having mapped out the learner's context, we begin an iterative participatory process of design with the aim of developing technology that facilitates access to appropriate resources at appropriate times during the learning process. The EoR has been used with learners and teachers across a range of subjects, including science and language learning to design technology rich learning activities and technology applications, such as smart phone applications (Underwood, Luckin, \& Winters, 2011).

\section{The Ecology of Resources Design Process}

The EoR design process offers a three-phase structure through which educators and technologists can work together to develop technologies and technology-rich learning activities that take a learner's wider context into account. These phases entail examining and developing a model of the learner's context. The phases consist of the following activities:

Phase 1: Create an Ecology of Resources Model to identify and organize potential resources for learning.

Phase 2: Identify the relationships within and between the resources produced in Phase 1 . Identify the extent to which these relationships meet a learner's needs and how they might be optimized.

Phase 3: Develop the Scaffolds and Adjustments to support learning and enable the negotiation of a Zone of Proximal Adjustment (ZPA) for a learner (Luckin, 2010). This 
consists of identifying the possible ways in which the relationships identified in Phase 2 might best be supported or scaffolded. This support might, for example, be offered through the manner in which technology is introduced, used or designed.

The study reported here is part of a research project to design technologies that support teenagers to learn about energy consumption and energy related issues, and encourage changes in personal energy consumption. In this paper, our focus is on Phase 1 of the EoR (see Luckin, 2010 for framework detail), which comprises six iterative steps to support the modelling process. Some steps require several iterations. Step 2 is of particular importance and can require several iterations through the other steps. The steps in Phase 1 of the EoR and the project-specific associated research questions are:

Step 1 - Brainstorming Potential World Resources

a. What are the circumstances in which teenagers use energy?

b. What energy consumption is most relevant to them?

c. What are their sources of information about energy consumption and energy sustainability?

Step 2 - Specifying the Focus of Attention

This is informed by three elements: a) the current body of knowledge about energy consumption and energy sustainability issues, derived from the literature, for example, the energy intensity of various behaviours, and environmental impact of various sources of energy, b) the energy consumption that is relevant to teenagers (Step 1 of Phase 1), and c) teenagers' current understanding about energy consumption and energy sustainability (Step 5 of Phase 1). The EoR design process is iterative. Step 2 emerges gradually through the entirety of empirical work in Phase 1. 
Step 3 - Categorizing World Resource Elements from Step 1 into three categories: Knowledge and Skills, People and Tools, Environment.

Step 4 - Identify potential Resource Filters

What are the constraints on:

a. information available to teenagers about energy consumption and energy sustainability?

b. the choices teenagers have on the energy they use?

c. how visible energy consumption is to teenagers?

Step 5 - Identify the Learner's Personal Resources

(conceptions of energy issues)

a. How do teenagers understand energy consumption and energy sustainability in their everyday life?

b. How aware are they of their energy consumption?

c. How much knowledge do they have about the energy intensity of their behaviour and choices?

(concerns and motivations)

d. Are teenagers concerned about energy sustainability?

e. What are their attitudes towards energy saving?

f. What motivates them to save energy?

Step 6 - Identify potential More Able Partners (MAPs).

a. Who are the potential more able partners in relation to understanding energy consumption and energy sustainability?

b. Who are the potential more able partners in relation to motivating to learn about energy consumption and energy sustainability? 


\section{Method}

We report here on Phase 1 of the EoR design process. Phase 1 requires the application of appropriate methods to identify and model key resources in learners' context. This is composed of six steps through which an EoR model of learners' context is created. The details of these six steps and the investigative methods we used are described below.

\section{Participants}

We worked with two groups of participants. Group 1 included 14 teenagers (aged 14-17; 5 female and 9 male) and Group 2 included 29 participants (aged 14-17; 6 female and 23 male). Both groups were enrolled in the same IT course at an international school in the UK. The sample that participated in this study is not representative of the teenage population. However, their conceptions of energy consumption and motivations are still very relevant as this cohort come from families with higher incomes. They are therefore likely to be greater consumers of energy (Abrahamse \& Steg, 2011), both now and in the future.

\section{Procedure}

\section{Group 1}

The course teacher integrated our research with a course project, which was completed shortly after the study. This allowed us to work with the students over 3 sessions and also to gather data from a student-led presentation of their formally assessed work. We used a combination of photo diaries and focus groups to explore the research questions.

Photo diaries. Participants were asked to create a photo diary of a day in their lives. We did not want to prescribe the kinds of things the participants should identify as relevant to energy and we did not provide reminders, because we were interested in what activities the 
participants would choose to document. Instead we asked them to document any change of activity that they noted during the day and we suggested that they aim to take 24 photos. We also dedicated a separate session to discussing the ethical reasons for maintaining anonymity when taking photos of people. We set up the photo diary activity on a Friday and the participants completed it between Friday afternoon and Saturday, thereby capturing activities both in school and at the weekend.

Focus groups. The photos from the diaries were revisited 2 days after they were created. Participants worked in groups of 3-4 and took turns in creating a narrative from their photos, by laying them out as a storyboard with captions. Within the group each teenager was assigned a role of interviewee (the person whose diary was being discussed), interviewer(s), or note-taker. The interviewer(s) were given the following questions as a guide: a) What were you doing when you took the photo? b) What else was going on around you? c) How long were you doing this activity for? and d) Why did this activity end?

Once the narrative was complete we discussed the activities that were captured in the photos through the lens of energy consumption. For this discussion we provided the interviewer(s) with the following questions: a) Is what you were doing related to energy use? If so, in what way is it related to energy? Where you conscious of how much energy you were using? Could you use less energy? Would you choose to use less energy? b) Are there times when you intentionally use less energy? If so, when? c) Are you concerned about energy use?

Formal presentation and small group discussion. After the photo diary sessions the students completed a formally assessed project on how they could reduce their energy consumption. They visited the university department to present their work to our colleagues. After a general post-presentation discussion we divided the participants into discussion groups of 4-5. One of 
the researchers led each discussion. We used some of the participants' photos as prompts to probe their conceptions further, focusing in particular on indirect use of energy (that is, energy used through the consumption of products and services). We had identified this as a key issue about which participants appeared to have less awareness, based upon a preliminary analysis of the photo diaries and focus groups. To seed these discussions we used photos of a bottle of water, filtered water, a zero-sugar soft drink, food (a burger), and clothes, taken by participants.

Feedback session. Following our analysis of the data (as discussed in the following section), we arranged a focus group to obtain feedback from participants about our analysis. Five of the 14 original participants attended. During this session we discussed our findings, primarily regarding their personal resources (that is, their conceptions, concerns and motivations about energy). We used questionnaires to gather data and structure the discussion. In the questionnaires we asked them (a) to rate their understanding of how they use energy, and their understanding of how they can save energy by placing a mark on a continuous scale, and (b) to complete a modified ecological footprint questionnaire. This acted as a prompt for respondents to consider the range of behaviours that have an environmental impact related to energy (including both behaviours they had and had not considered in the previous study; this questionnaire was modified from Chauhan et al., 2010 to include items relevant to energy).

\section{Group 2}

The larger number of participants and time constraints required a 'lighter touch' approach with this group. We therefore used focus groups and a questionnaire to collect data. 
Focus group. We created a list of statements to enquire into participants' understanding of energy consumption, their concerns and motivations. For each statement we asked the participants to individually rate the extent to which they agreed with the statement (on a continuous scale). We then discussed each rating, as a group. The statements were:
A. Energy use is a severe and urgent problem
B. I understand why energy use is a problem
C. Energy use is a problem that will affect myself and those close to me
D. I am concerned about the energy problem
E. I am aware how I contribute to energy use (overall, not just household bills)
F. I have a clear understanding of what needs to be done to address the problem
G. I want to have a good understanding of the problems to do with energy
H. I know how to learn more about the problems to do with energy
I. I am part of the solution to the problem
J. I am aware of everything I can do to save energy (overall, not just the household bills)
$\mathrm{K}$. It is my responsibility to address the problem of energy use

To further explore concerns about energy issues we asked participants to complete the following task: (i) draw three lines listing the people, places and things that are important to you (along a continuum starting with the most important on the left); and (ii) mark the people, places and things that would be affected by issues around energy (see Figure 1). We then discussed how the participants believed they would be affected.

[insert Figure 1 here]

Post focus group questionnaire. A week after the focus group the participants completed a questionnaire. Our aim was to capture their views after they had explored the ideas discussed 
in the focus group. In the first part of the questionnaire they were asked (i) to list the ways in which they use the most energy, (ii) the ways in which they can save the most energy, (iii) whether they think we will need to change our lifestyles in the future (because of energy problems) and if so (iv) what we will need to change. In the second part of the questionnaire we created a list of barriers to saving energy drawing on our data from Group 1 and from the literature. The participants were asked to respond to several statements about these barriers, indicating whether each barrier applied to them (on the scale: 'not at all true', 'not true', 'true', 'very true', 'don't know'). The statements were:
A. I don't know/understand what the problem about energy is
B. I don't know what I can do to save energy
C. I don't believe it's something I personally need to do anything about
D. I don't believe it's something I personally can do anything about
E. I don't have much choice in what energy I use
F. It's habit, I don't even notice how much energy I'm using
G. It's too much effort to save energy
H. It's not my responsibility to save energy

I. Nothing I do will make much difference

J. I think that changing my behaviour to save energy would make my life less enjoyable

$\mathrm{K}$. Saving energy is not what everyone else is doing, so I'm not either

L. I don't want to be seen as an eco-warrior, so I don't do anything to save energy

M. I think the problem about energy is not that severe so I'm not doing anything about it

N. I'm not concerned about the problems around energy use

O. I don't believe the problems about energy will affect me, so I don't do anything about it 


\section{Analysis and Findings}

We report on the results of Phase 1 of the EoR. Phase 1 involves identifying the resources that make up the learners' contexts in relation to energy consumption and forming an understanding of the learner and their personal resources.

We collected 3 sets of data: (i) from our first engagement with Group 1 (photo diaries, focus group discussion, formal presentation, and small group discussion audio recordings), (ii) from the feedback session with Group 1 (group discussion audio recording), and (iii) from Group 2 (group discussion audio recording and questionnaires).

We analysed the data by an iterative process of identifying themes. We identified and extracted those conceptions, motivations and context elements that recurred across participants and across data sources (photo diaries, focus group discussions, formal presentation, small group discussions, and questionnaires). For example, in relation to Step 1, question (a) "What are the circumstances in which teenagers use energy?", we identified the instances of energy consumption that recurred across all data sources within the data set. Through this process we identified the themes that occurred most frequently across all data sets. We used a scale from 1 to 7 to analyse the questionnaire responses on the continuous scales. For the analysis of the barriers questionnaire, we used a scale from 1 to 4 , where 'not at all true' is 1 and 'very true' is 4.

The analysis from Group 1 supported construction of an initial EoR model, which was later refined with the addition of data from the feedback session with Group 1 and the data from Group 2. Step 2 is discussed at the end in order to place it in the contexts of steps 1-6. We have structured our findings based on Steps $1-6$ of Phase 1 of the EoR and the research questions. 


\section{Steps 1 \& 3: Identifying and categorising world resources}

Our focus in addressing this question was on the kind of activities the participants engage in and the choices they make. We analysed the data (viz., photo diaries, focus sessions, formal presentation, small group discussions, and questionnaires) by identifying all the activities, contexts of use, people and products, within the data set. We then selected those items that occurred with the greatest frequency, grouping them into the EoR categories: Knowledge and Skills, People and Tools, and the learner's Environment.

People and Tools. What energy artefacts are most relevant to teenagers?

We identified three groups of energy artefacts that are relevant to the majority of our participants: electronic devices, food, and also personal care products. Although there was individual variation within each category, there were some common products across participants: computers, phones, music players, drinks (energy drinks), and water (bottled, filtered, tap).

Environment. What are the environments in which teenagers use energy?

The home was the environment that appeared across all photo diaries, formal presentations, focus group discussions, and questionnaires around energy consumption. School and travel from home to school also featured but in fewer than half the instances of energy use. Knowledge and Skills. What are teenagers' sources of information about energy and energy saving?

The source of information the majority of participants mentioned explicitly, and that was brought up in discussions of energy, were their parents. Parents appeared to play a role in relation to not wasting energy. In terms of facts about energy consumption and energy intensity of behaviours, a few participants mentioned teachers, TV programs and online information. For example, one participant mentioned a program about the amount of energy that can be saved through recycling. It is worth noting that in all cases when information from 
TV programs was mentioned it was presented with uncertainty as something that they had heard but couldn't quite recall the details of.

\section{Step 4: Filters}

We analysed the data to identify filters that impact on the learners' access to the world resources (see Steps $1 \& 3$ ). The filters we identified are based on our interpretation of teenagers' discussions on their energy use and its impact. Filters to the participants' energy consumption appeared to be the visibility of electricity use, the lack of available information, and that teenagers do not always make the decisions on what to buy (for example, in relation to food or personal care products). Their parents' knowledge and motivation to save energy and to discuss it also appeared to act as filters to learning about energy consumption. Having enough knowledge to understand about energy from online resources, the scarcity of personalised information on energy consumption that is tailored to teenagers' circumstances and motivations, and the presence of the skills and motivation to search for information were also identified as filters relevant to learning about energy.

\section{Step 5: Learner Resources}

Teenagers' resources: conceptions of energy issues

How do teenagers understand energy consumption and saving in their everyday life? The discussions of energy mostly concerned electricity and the use of electronic devices (that is, direct energy consumption). This also was evident in the participants' formal presentations, most of which focused exclusively on electricity. The only other forms of energy consumption that were mentioned were heating water (2/14 participants in Group 1 and 6/29 in Group 2), transport (3/29 participants in Group 2) and one participant in Group 1 mentioned energy required to produce plastic water bottles and paper. 
This focus on direct energy consumption and lack of awareness of indirect energy consumption is interesting principally because when prompted about indirect energy consumption explicitly participants engaged in discussion about it. In the small group discussions with Group 1 (after the formal presentation), we prompted participants with selected photos of food, drink, and clothes, with the aim of eliciting their conceptions about indirect energy consumption. We had identified this as an area of low awareness from the photo diaries and focus groups. During the discussion participants discussed several factors contributing to energy consumption including production, transportation, refrigeration (in the case of food and drink), packaging, and waste processing of products. When prompted further they also discussed the relative energy intensity of different foods (for example, meat versus vegetarian products). Similarly, when we prompted discussion about indirect energy consumption with Group 2 participants mentioned various factors including production, transport and waste processing of products.

Participants also focused on energy consumption that is solely due to their behaviour. All the examples of energy consumption and saving discussed by both groups, and those reported in the questionnaire by Group 2 related to individual (rather than shared) energy consumption. However, when prompted they did discuss shared use of energy. For example, each photo diary contained several pictures of food. During the discussions the participants were prompted to consider what uses of energy are associated with, for example, their dinner, which led to a discussion about the energy consumption associated with dishwashers, microwave ovens, and refrigeration.

Participants' ideas for saving energy were limited to using less, for example, switching off devices when not in use, or using their laptop less. They did not consider consuming different products or engaging in alternative activities. This was the case for both Groups 1 and 2. The responses to the questionnaire (Group 2) on ways to save energy 
included using less energy not finding alternatives, with the exception of using alternative transport (4/29 participants).

It appears the concept of energy and energy saving is closely tied with not wasting more generally, and it is closely linked with not wasting other resources. For example, during the photo diary discussions many participants mentioned water (though not heating water) and compared not wasting electricity with not wasting water. When asked specifically about food during the small group discussions, the focus for many was again on waste. Similarly, with regard to clothes the focus was on handing them down to others, rather than discarding them. From the focus groups it emerged that this cohort are prompted by their parents to not waste electricity and water, and many are given their siblings' clothes, and so it is not surprising that these are closely linked ideas.

How aware are teenagers of their energy consumption? Almost all participants reported they were generally not aware of their energy consumption prior to engaging with us. Their teacher also confirmed the change in their awareness over the course of the study. The only instance they discussed of being aware of energy consumption and acting to save energy was switching off lights and music players when they left the room, as prompted to by their parents. We note that while they were able to discuss the different uses of energy, and even indirect uses when asked explicitly about them, this information was not translated into awareness of personal energy consumption in their everyday life until we prompted them to reflect on it.

How much knowledge do teenagers have about the energy intensity of their behaviour? The participants' discussions of how they could save the most energy indicated little awareness of the energy intensity of different behaviours. They appeared to base their calculations of the 
amount of energy consumed by how long a device had been switched on. For example, because a fridge or phone is continuously in use it was assumed to use more energy. Discussions around indirect energy consumption suggested that, while they were aware of indirect energy requirements, they considered it negligible in comparison with energy consumed by lights or electronic devices.

It is interesting to note that, in rating their understanding about energy and energy related problems in the focus group questionnaire, Group 2 reported they are knowledgeable and are motivated to learn (see items B, E, G and $\mathrm{H}$ in Table 1). They were slightly less sure of what the solutions to the problems are and what they can do to save energy (see items F and $\mathrm{J}$ in Table 1), though these ratings are still relatively high.

[insert Table 1 here]

Teenagers' resources: concerns and motivations

Are teenagers concerned about energy issues? There was agreement across both groups that energy related problems are important and relatively urgent issues. The problems discussed included climate change, resource scarcity, and animal habitat destruction. Group 2 also reported in the questionnaire that they consider energy problems to be important, though they reported a lesser degree of concern (see items A, C and D in Table 1). The participants did not express great concern during the discussions and were vague about how they might be affected. During the activity on how energy problems will affect them (see Figure 1), the participants in Group 2 indicated their main concern is the availability of energy. Five mentioned food and water supply as another way in which they may be affected. Although during discussion a few participants suggested or agreed that problems would be resolved through improved technology, the majority did not appear to have reflected on the issues and did not elaborate on their concern. 
What are teenagers' attitudes towards energy saving? The focus group discussions indicated that for the majority of participants the responsibility of alleviating the problems around energy consumption does not lie with them. Their perception was that this is not something they should be expected to take responsibility for. However, the majority indicated a willingness to take responsibility for individual actions and stated that each person should lead by example. This was also reflected in the questionnaire responses of Group 2. They indicated they should play their part, but addressing energy related problems is not their responsibility (see items I and $\mathrm{K}$ in Table 1). This finding is not surprising but it highlights an interesting issue in perception of responsibility more generally. For these teenagers the question of whether they should to act appeared to be about it being unreasonable for adults to expect them to act. It was not seen as a problem that affects them and, consequently, one that they might choose to do something about.

When we discussed their willingness to change their behaviour in order to save energy, the majority seemed very willing to act to prevent waste but not to modify their lifestyle. They also doubted that anyone else would be open to lifestyle changes. Their focus was only on limiting amount of use rather than finding alternatives. In relation to indirect energy consumption, as we have discussed, our cohort of teenagers did not consider indirect energy consumption to have as great a social impact, so they did not discuss the possibility purchasing alternative products or services. When queried about what factors would affect their purchases their focus was overwhelmingly on financial cost, and to a lesser degree, on the impact on people. For example, they all agreed that they would not buy clothes produced by child labour. 
The responses from Group 2 on the post-study questionnaire indicated that the participants perceived habit (item F) as a marginally stronger barrier to saving energy (see Table 2).

[insert Table 2 here]

What motivates teenagers to save energy? None of the participants reported that they consciously change behaviour to save energy. The main motivation was their parents' telling them to switch off the lights and electronic devices when not in use.

\section{Step 6: More Able Partners}

We identified the potential More Able Partners (MAP) in the learners' contexts that might play a role in scaffolding learning. For these groups of teenagers parents were the main potential MAP. School, friends, and online resources were also identified as MAP across focus group discussions with both groups 1 and 2 . The potential of each partner to support the learner is shaped by the nature of the learner's interaction with them and the knowledge available to the learner from them. The relationships between the learner and more able partners will be explored further in Phase 2 of the EoR, which involves identifying and modelling the connections between the resources in the learners' contexts.

\section{Step 2: Focus of Attention}

Awareness of indirect energy consumption and knowledge of the relative energy intensity of different behaviours emerged as important areas for learning. The energy consumption that appears to be most relevant to teenagers is that involved in electronic devices (primarily computers and phones), food, drink, and personal care products. Also participants' focus on individual energy consumption suggests we need to make more salient their contribution to shared (within the family) and public energy consumption. The critical challenge is to motivate teenagers to engage with issues around energy consumption. Although they report 
concerns, it seems the problems around energy consumption are too abstract for them to make a connection with their lives and everyday behaviour (as appears to be the case with adults, Lorenzoni et al., 2007). The priority is to foster understanding of the impact of energy issues on people, both those close to them and those further away.

\section{Discussion}

The study we report in this paper is part of ongoing research to design technology to support teenagers' learning about energy issues and the impact of their individual behaviour and choices. We engaged with 2 groups of teenagers using mainly qualitative methods (photo diary, focus groups and formal project work). Our aim was to identify important areas of focus for learning, to understand how to motivate teenagers to engage in learning about energy, and to explore how the technology can be integrated into learners' context, thereby linking the learning material and the (everyday) context in which it applies. The design of learning technologies must tap into learners' motivations and the multiple influences and resources within the learners' contexts. The methodology needs to be participatory to ensure that a clear and accurate understanding of the learners' personal contexts is integrated within any resultant design. The EoR methodology that we applied offers a process for working with participants that models and takes this into account.

We report on Phase 1 of the EoR, which involves identifying and modelling learners' contexts. Phase 1 forms the basis on which, in Phases 2 and 3, technology is designed to scaffold learning by supporting appropriate connections between the resources in a learner's context. The current work contributes to understanding of: a) more generally, methods (within the context of the EoR) for engaging with teenagers to identify and model their personal contexts, and b) specifically, the design of learning technologies to support teenagers to learn about personal energy consumption. 


\section{Research methods to model personal context}

In this paper we have presented the application of Phase 1 of the EoR in the context of designing technology to support teenagers to learn about personal energy consumption. We discuss the issues around the research methods we used to identify and model teenagers' personal contexts.

We purposely used open-ended tasks that did not focus, at the outset, on energy. This was important in understanding the participants' informal contexts as filtered by what they considered relevant to their lives and also acceptable to share with us. The data also confirmed the importance of an open-ended method for exploring teenagers' awareness in the context of their everyday lives and differentiating awareness and knowledge in this context from the more formal knowledge they have about energy. For example, when we discussed their photo diaries indirect energy consumption was hardly touched upon. However, when we used selected photos from the diaries to prompt them explicitly about indirect energy consumption related to food and water we generated a discussion around energy requirements for production, transportation, storage, and disposal. Had we only conducted a focus group around examples of indirect energy consumption we might have assumed this information would be readily available at all times.

The photos from the diaries also allowed us to centre the discussion on instances of energy consumption that were personally relevant and, therefore, more engaging. Energy consumption is not visible and, aside from the cost of energy bills, most individuals in the UK do not directly feel the impact of energy related issues. Therefore, to make the discussion around energy less abstract and more personally relevant it was important to use examples from the participants' lives. For example, instead of discussing the energy cost of producing meat vs. vegetables, we could discuss the energy cost implied by a picture of a hamburger, taken by one of the participants. 
When we asked participants to create a photo diary of a day in their lives we did not predisposed them to consider energy consumption. Given our finding that they were largely unaware of indirect energy consumption, this was crucial for rich data collection. Had we set up the task explicitly to capture energy consumption, or had we held a focus group on the topic of energy consumption without the photo diaries, the teenagers would probably not have captured or mentioned any activities that did not explicitly involve electricity use. We would, therefore, not have had any data on the indirect energy consumption that is relevant to teenagers.

Participatory design is an important part of the EoR process. Through this participatory process, we ensure that the learning technologies we develop will focus on the resources that are most relevant and engaging to learners. Thus when we discussed our findings with a subset of Group 1 we were able to confirm our interpretation of their conceptions, concerns and motivations around energy consumption.

The collection and discussion of the photo diaries was time intensive. Once we had gathered data from Group 1 we had a preliminary understanding of the participants' contexts. Therefore, in our subsequent engagement with Group 2 we used this as a basis on which to run a less time intensive focus group and questionnaire study, while still tailoring the examples and discussion to our teenage population.

The combination of multiple sources of data was also important to our method. The photo diary and subsequent focus group sessions allowed us to probe into the learners' informal contexts and the nature of their conceptions within those contexts. The students' assessed formal project work regarding their energy consumption and the ways they could save energy gave us an insight into their formalised notions of energy consumption, especially as this assignment was conducted after the diaries and focus groups. When we started the study they had not yet explored their energy consumption habits so were still at 
their pre-study awareness level. When they began the formal project work they had already engaged in discussions about energy use with us. Therefore, it is reasonable to assume that what was distilled in their formal work represented core ideas (for example, the focus on direct energy over indirect, and their perception of the relative energy intensity of different activities).

\section{Implications for technology design}

In this paper we have reported research on Phase 1 of the EoR which has resulted in a model of teenagers' personal contexts in relation to their learning about energy. These findings have implications for the design of personalised technology to support learning about personal energy consumption (which will be developed during work in subsequent phases of the EoR, with further participation from teenagers). We summarise the most significant findings.

Personalised learning technologies need to focus on learning that is closely tied to teenagers' current understanding and to energy consumption that is relevant to teenagers. The critical issues about which there appears to be a lack of awareness amongst teenagers are: the impact of energy related problems, the energy consumption involved in products (indirect energy consumption), and how much energy is required by different electronic devices. Our findings also identified the following energy consumption as most relevant to teenagers: a) direct energy consumption: energy consumed individually through use of electronic devices, particularly computers and phones, and b) indirect energy consumption: food (and drink), personal care products, and buying electronic devices.

The present findings also contribute to our understanding of what resources are already available to teenagers, that learning technologies can be designed to tap into. Teachers appear to be best informed to mediate learning about energy, but parents may be better placed to influence behaviour (at least in relation to energy consumption that parents are conscious of, such as switching off lights). Online and other media resources are 
available, but their impact on teenagers' awareness and behaviour does not appear to be significant. Learning technologies must support teenagers access and translate the information from media resources to their personal context.

Critically for the design of any learning technology, motivation to engage in learning about energy cannot be assumed. In fact, our findings strongly suggest that, while teenagers view energy as an important issue, they are not particularly concerned about it and do not demonstrate motivation to learn how to reduce their impact. However, this cohort of teenagers also appeared to be unaware of how energy issues might impact on them and the rest of the world. Therefore, a first step in fostering motivation to learn might be to make this impact more concrete and personally relevant.

\section{Study Limitations and Ongoing Work}

We worked with a small number of participants, which allowed us to collect detailed data, but also limited the generalisability of our findings. However, the fact that our results largely replicate research reports with adults in terms of awareness of energy consumption and motivation to reduce it (Lorenzoni et al., 2007; Steg, 2008; Whitmarsh, 2009), suggests that the issues we have identified as important generalise beyond our sample. Planned work with different groups of teenagers will contribute to the EoR model. In subsequent studies we will compare our current findings on teenagers' personal context (as modelled by the EoR) with those from teenagers from a range of schools and backgrounds. Future work in Phases 2 and 3 will prototype learning technology based on these findings on teenagers' personal contexts, and will further involve teenagers in a participatory design process. 


\section{Acknowledgements}

We thank the students and staff who have worked with us on the Taking in the Teenagers project funded by the Engineering and Physical Sciences Research Council in the UK: Grant reference number EP/I000550/1.

\section{References}

Abrahamse, W., \& Steg, L. (2011). Factors Related to Household Energy Use and Intention to Reduce It: The Role of Psychological and Socio-Demographic Variables. Human Ecology Review, 18(1), 30-40.

BBC (2006). Teenagers are 'standby villains'. Available online at:

http://news.bbc.co.uk/1/hi/scotland/6219862.stm (Accessed 1 November 2011).

Chauhan, S., Rama das, S., Haigh, M., \& Rita, N. (2010). Awareness vs intentionality: exploring education for sustainable development in a British Hindu community. Sustainable Development, 20(6), 361-371.

Larsson, B., Andersson, M., \& Osbeck, C. (2010). Bringing Environmentalism Home: Children's influence on family consumption in the Nordic countries and beyond. Childhood, 17(1), 129-147.

Lorenzoni, I., Nicholsoncole, S., \& Whitmarsh, L. (2007). Barriers perceived to engaging with climate change among the UK public and their policy implications. Global Environmental Change, 17(3-4), 445-459.

Luckin, R. (2010). Re-designing Learning Contexts: Technology-Rich, Learner-Centred Ecologies. London and New York: Routledge.

Luckin, R., Looi, C.K., Puntambekar, S., Stanton Fraser, D. Tabak, I. Underwood, J., \& Chen W. (2011) Contextualizing the changing face of Scaffolding Research: Are we driving pedagogical theory development or avoiding it? Proceedings of the International 
Conference of Computer Supported Collaborative Learning, Hong Kong. 1037 1045.

Pea, R.D. (2004) The social and technological dimensions of scaffolding and related theoretical concepts for learning, education, and human activity. Journal of the Learning Sciences, 13, 423-51.

Steg, L. (2008). Promoting household energy conservation. Energy Policy, 36, 4449-4453.

Thøgersen, J., \& Grønhøj, A. (2010). Electricity saving in households: A social cognitive approach. Energy Policy, 38(12), 7732-7743.

Underwood, J., Luckin, R. \& Winters, N. (2011). Retelling Stories: Setting Learner Narratives in Resource Ecologies. Proceedings of the International Conference of Computer Supported Collaborative Learning, Hong Long. pp 611 - 616.

Vygotsky, L. S. (1978). Mind in Society: The Development of Higher Psychological Processes. Trans. Cole, M., John-Steiner, V., Scribner, S. \& Souberman, E. Cambridge, MA: Harvard University Press.

Vygotsky, L. S. (1986). Thought and Language. Cambridge, MA: MIT Press.

Wood, D. J., Bruner, J. S. \& Ross, G. (1976) The Role of Tutoring in Problem Solving. Journal of Child Psychology and Psychiatry, 17(2), 89-100.

Whitmarsh, L. (2009). Behavioural responses to climate change: Asymmetry of intentions and impacts. Journal of Environmental Psychology, 29(1), 13-23. 